\title{
Counteracting neuronal nitric oxide synthase proteasomal degradation improves glucose transport in insulin-resistant skeletal muscle from Zucker $f a / f a$ rats
}

\author{
Karima Mezghenna • Jérémy Leroy • Jacqueline Azay-Milhau • \\ Didier Tousch • Françoise Castex • Sylvain Gervais • \\ Viviana Delgado-Betancourt • René Gross • Anne-Dominique Lajoix
}

Received: 11 March 2013 / Accepted: 30 September 2013 / Published online: 2 November 2013

(C) Springer-Verlag Berlin Heidelberg 2013

\begin{abstract}
Aims/hypothesis Insulin-mediated glucose transport and utilisation are decreased in skeletal muscle from type 2 diabetic and glucose-intolerant individuals because of alterations in insulin receptor signalling, GLUT4 translocation to the plasma membrane and microvascular blood flow. Catalytic activity of the muscle-specific isoform of neuronal nitric oxide synthase (nNOS) also participates in the regulation of glucose transport and appears to be decreased in a relevant animal model of drastic insulin resistance, the obese Zucker $f a / f a$ rat. Our objective was to determine the molecular mechanisms involved in this defect.

Methods Isolated rat muscles and primary cultures of myocytes were used for western blot analysis of protein expression, immunohistochemistry, glucose uptake measurements and GLUT4 translocation assays.

Results nNOS expression was reduced in skeletal muscle from $f a / f a$ rats. This was caused by increased ubiquitination of the enzyme and subsequent degradation by the ubiquitin proteasome pathway. The degradation occurred through a greater interaction of nNOS with the chaperone heat-shock protein 70 and the co-chaperone, carboxyl terminus of Hsc70interacting protein (CHIP). In addition, an alteration in nNOS sarcolemmal localisation was observed. We confirmed the implication of nNOS breakdown in defective insulin-
\end{abstract}

Electronic supplementary material The online version of this article (doi:10.1007/s00125-013-3084-9) contains peer-reviewed but unedited supplementary material, which is available to authorised users.

K. Mezghenna $\cdot$ J. Leroy $\cdot$ J. Azay-Milhau $\cdot$ D. Tousch $\cdot$ F. Castex $\cdot$

S. Gervais $\cdot$ V. Delgado-Betancourt $\cdot$ R. Gross $\cdot$ A.-D. Lajoix $(\square)$

Centre for Pharmacology and Innovation in Diabetes, University

Montpellier 1, EA 7288, 15 Avenue Charles Flahault, BP 14491,

34093 Montpellier cedex 5, France

e-mail: anne-dominique.lajoix@univ-montp1.fr induced glucose transport by demonstrating that blockade of proteasomal degradation or overexpression of nNOS improved basal and/or insulin-stimulated glucose uptake and GLUT4 translocation in primary cultures of insulin-resistant myocytes.

Conclusions/interpretation Recovery of nNOS in insulinresistant muscles should be considered a potential new approach to address insulin resistance.

Keywords Insulin resistance $\cdot$ NO synthase - Obesity · Skeletal muscle $\cdot$ Type 2 diabetes

$\begin{array}{ll}\text { Abbreviations } \\ \text { CHIP } & \text { Carboxyl terminus of Hsc70-interacting protein } \\ \text { eNOS } & \text { Endothelial nitric oxide synthase } \\ \text { Hsp } & \text { Heat-shock protein } \\ \text { nNOS } & \text { Neuronal nitric oxide synthase } \\ \text { NO } & \text { Nitric oxide } \\ \text { NOS } & \text { Nitric oxide synthase } \\ \text { PIN } & \text { Protein inhibitor of neuronal nitric oxide synthase } \\ \text { SNP } & \text { Sodium nitroprusside } \\ \text { ZDF } & \text { Zucker diabetic fatty }\end{array}$

\section{Introduction}

Nitric oxide (NO) is a short-lived gas produced by a family of enzymes called NO synthases (NOSs). Three isoforms have been identified, including constitutive neuronal (n)NOS, endothelial (e)NOS and cytokine-inducible NOS (iNOS) [1]. Human and rodent skeletal muscle fibres mainly express nNOS [2], whereas eNOS, which is also present in some muscle fibres in rodents [3], is predominantly found in the endothelium of blood vessels within human skeletal muscle 
[2]. A unique nNOS isoform, $\mathrm{nNOS} \mu$, a splice variant of nNOS $\alpha$ that displays an insertion of 34 amino acids between exons 16 and 17 without any modification of its catalytic activity, is expressed in skeletal muscle [4]. nNOS is mainly but not exclusively distributed along the sarcolemma of positive fibres [2], as a component of the dystrophin glycoprotein complex [5].

nNOS is involved in the regulation of contractile force, insulin- and exercise-stimulated glucose transport, energy metabolism and blood flow (for review, see [6] and [7]). Interestingly, the nNOS signalling pathway is altered in various pathologies including Duchenne muscular dystrophy and type 2 diabetes. Indeed, type 2 diabetes is characterised by both pancreatic beta cell secretory defect and insulin resistance of the peripheral tissues and liver. Skeletal muscle displays decreased insulin-mediated glucose transport and utilisation in type 2 diabetic patients, as well as in those with glucose intolerance [8], as a consequence of impaired insulin signalling and GLUT-4 translocation pathways (for review, see [9]).

The demonstration of a role for nNOS in insulin resistance emerged from a study by Shankar et al [10], which showed that nNOS-knockout mice were insulin resistant at the level of peripheral tissues. In addition, Young and Leighton provided evidence for the possible involvement of decreased NOS catalytic activity in abnormal glucose transport and utilisation in the insulin-resistant Zucker fa/fa rat [11]. The aim of this study is to investigate the molecular mechanisms involved in the alteration of NOS catalytic activity observed in the Zucker $f a / f a$ rat. Zucker $f a / f a$ rats display a mutation in the leptin receptor [12], with hyperphagia, obesity, insulin resistance and hyperinsulinaemia as phenotypic characteristics. We found that nNOS expression was reduced in the skeletal muscle of $f a / f a$ rats because of increased degradation of the enzyme by the ubiquitin-proteasome pathway. Importantly, restoration of nNOS expression and catalytic activity improved glucose uptake and GLUT4 translocation in insulin-resistant myocytes.

\section{Methods}

Animals Male Zucker fa/fa rats and their control littermates, $\mathrm{fa} /+$ rats, were purchased from Harlan (Blackthorn, UK) and Zucker diabetic fatty (ZDF) rats were purchased from Charles River (L'Arbresle, France) (see Electronic Supplementary Material [ESM] Methods). Institutional guidelines for animal care were followed and the protocol was approved by our University ethical committee. Zucker $f a / f a$ rats were killed at the age of 9-11 weeks and ZDF rats were killed at the age of 10 weeks.

Real-time reverse transcription (RT)-PCR Total RNA from gastrocnemius muscles was extracted with RNA Now reagent
(Biogentex, League City, TX, USA). First-strand cDNA was obtained from $2 \mu \mathrm{g}$ total RNA using SuperScript II RNAse $\mathrm{H}^{-}$ Reverse Transcriptase (Invitrogen, Paisley, UK). Real-time quantitative PCR was carried out to assess the gene expression of $n N o s$ (otherwise known as Nos 1 ), protein inhibitor of nNOS (Pin; otherwise known as Dynll1) and Gadph (as an invariant housekeeping gene) using specific primers. PCR was performed with FastStart DNA Master SYBR Green I mix (Roche, Basel, Switzerland) on a LightCycler (Roche).

Western blotting and immunoprecipitation Sections from the middle of the muscle were homogenised in lysis buffer containing $1 \%$ Triton $\mathrm{X}-100,0.1 \%$ SDS and protease inhibitors ( $1 \mathrm{ml}$ per $100 \mathrm{mg}$ tissue; Roche) using a Polytron homogeniser (IKA, Stanfen, Germany).

Proteins $(75 \mu \mathrm{g})$ were separated on a $7.5 \%$ SDSpolyacrylamide gel for nNOS and on a $14 \%$ tricine polyacrylamide gel for PIN. Incubation with primary antibodies (see ESM Methods) was performed overnight. Signals were acquired with a Vilber Lourmat imager (Marne-la-Vallée, France) and quantified by GeneTools image analysis software (Syngene, Cambridge, UK). In relative quantification graphs, protein levels are expressed relative to $\alpha$-tubulin. Each experiment shown is representative of three independent western blots.

For subcellular fractionation, skeletal muscles were homogenised in buffer containing $20 \mathrm{mmol} / \mathrm{l}$ Tris ( $\mathrm{pH} 7.4$ ), $150 \mathrm{mmol} / 1 \mathrm{NaCl}$ and protease inhibitors. The supernatant fractions were centrifuged at $50,000 \mathrm{~g}$ and the membrane pellets were solubilised for $1 \mathrm{~h}$ at $4{ }^{\circ} \mathrm{C}$ in lysis buffer containing $1 \%$ Triton X-100, $0.5 \%$ Nonidet P-40 and $0.5 \%$ sodium deoxycholate.

For immunoprecipitation experiments, muscles were homogenised in lysis buffer containing 1\% Triton X-100, $0.5 \%$ Nonidet P- 40 , protease inhibitors and $N$-ethylmaleimide (Sigma-Aldrich, Steinheim, Germany). nNOS or carboxyl terminus of Hsc70-interacting protein (CHIP) was immunoprecipitated from a $2 \mathrm{mg}$ extract with polyclonal anti-nNOS (BD Biosciences, Franklin Lakes, NJ, USA) or polyclonal anti-CHIP (Santa Cruz Biotechnologies, Santa Cruz, CA, USA) antibody overnight and proteins were immunoblotted as described above.

Immunofluorescence Sections from gastrocnemius muscles ( $8 \mu \mathrm{m}$ thick) were fixed with $2 \%$ paraformaldehyde and immunostained overnight with anti-nNOS (EuroProxima, Arnhem, the Netherlands), anti-PIN (Santa Cruz) or anti- $\alpha 1$ syntrophin (Sigma-Aldrich) antibodies. Fluorescence was observed with the Zeiss (Oberkochen, Germany) LSM 780 confocal microscope using the Montpellier RIO Imaging platform (Montpellier, France). A negative control was performed by incubating sections with the secondary antibody. 
Incubation experiments with isolated muscles Each freshly isolated muscle was fixed on a plastic holder at its original length and placed vertically in an assay tube. After a $15 \mathrm{~min}$ preincubation period in Krebs-Henseleit bicarbonate buffer ( $\mathrm{pH}$ 7.4), muscles were incubated in the presence or absence of $50 \mu \mathrm{mol} / \mathrm{l} \mathrm{MG} 132$ (Sigma-Aldrich) for $2 \mathrm{~h}$ at $37^{\circ} \mathrm{C}$, with regular $95 \% \mathrm{O}_{2} / 5 \% \mathrm{CO}_{2}$ bubbling.

Isolation and culture of satellite cells Satellite cells were isolated from rat gastrocnemius muscles, as described in ESM Methods [13, 14]. For differentiation, myoblasts were seeded on 12-well coated plates in growth medium. After 5 days, the cells were incubated with DMEM containing $2 \%$ horse serum (differentiation medium) for another 5 day period.

Treatments of satellite cells Muscle cells were first differentiated for 3 days. For nNOS overexpression, an expression vector containing $n N o s \alpha$ cDNA (pCR3.1, Invitrogen) was introduced into muscle cells using PolyJet reagent (SignaGen, Ijamsville, MA, USA). Muscle cells were also treated for $48 \mathrm{~h}$ with $20 \mu \mathrm{mol} / \mathrm{l}$ cell-permeant ubiquitin proteasome system inhibitory Tat peptide, GRKKRRQRRRGGKAVDLSHQPS (EZBiolab, Carmel, IN, USA), or Tat alone, in control conditions [15]. nNOS expression was evaluated by western blot $48 \mathrm{~h}$ after each treatment and muscle cells were subjected to glucose uptake or GLUT4 translocation assay.

Glucose uptake in primary myocytes The day of the experiment, cells were first starved and then incubated for $1 \mathrm{~h}$ in KRB buffer, containing the compounds listed in the ESM Methods. [1,2- $\left.{ }^{3} \mathrm{H}\right]-2$-deoxyglucose uptake was performed for 5 min (see ESM Methods). Radioactivity was normalised to the total protein concentration $\left(\mathrm{cpm} \mathrm{mg} \mathrm{min}^{-1}\right.$ ). Changes are expressed in per cent of basal condition value (absence of insulin).

Translocation of GLUT4 Muscle cells were stimulated for $30 \mathrm{~min}$, fixed and incubated overnight with an anti-GLUT4 antibody (Alpha Diagnostic, San Antonio, TX, USA). Immunoreactivity was revealed with a peroxidase-conjugated antibody and a colorimetric reaction (see ESM Methods).

NOS catalytic activity assay NOS catalytic activity was estimated in muscle extracts by measuring the production of radiolabelled $\left[{ }^{3} \mathrm{H}\right]$ citrulline from $\left[{ }^{3} \mathrm{H}\right]$ arginine (MP Biomedicals, Irvine, CA, USA) according to the manufacturer's recommendation (Nitric Oxide Synthase Assay Kit, Merck Millipore, Billerica, MA, USA). NOS activity was measured after a $1 \mathrm{~h}$ reaction at $37^{\circ} \mathrm{C}$ and after separation of labelled citrulline from arginine by affinity chromatography.
Statistical analysis Experimental values represent the mean of $n$ experiments and are plotted on graphs as means \pm SEM. The data were analysed using a Student's $t$ test or ANOVA where applicable.

\section{Results}

Decreased nNOS expression in skeletal muscle from Zucker fa/fa rats Young and Leighton [11] previously reported decreased NOS catalytic activity in skeletal muscle from Zucker $f a / f a$ rats. We confirmed this defect in the insulinresistant gastrocnemius muscle, which displayed a $40 \%$ reduction in NOS catalytic activity $(p<0.01, n=4)$ (Fig. 1a). By western blot, we observed a $42 \%$ decrease in the nNOS
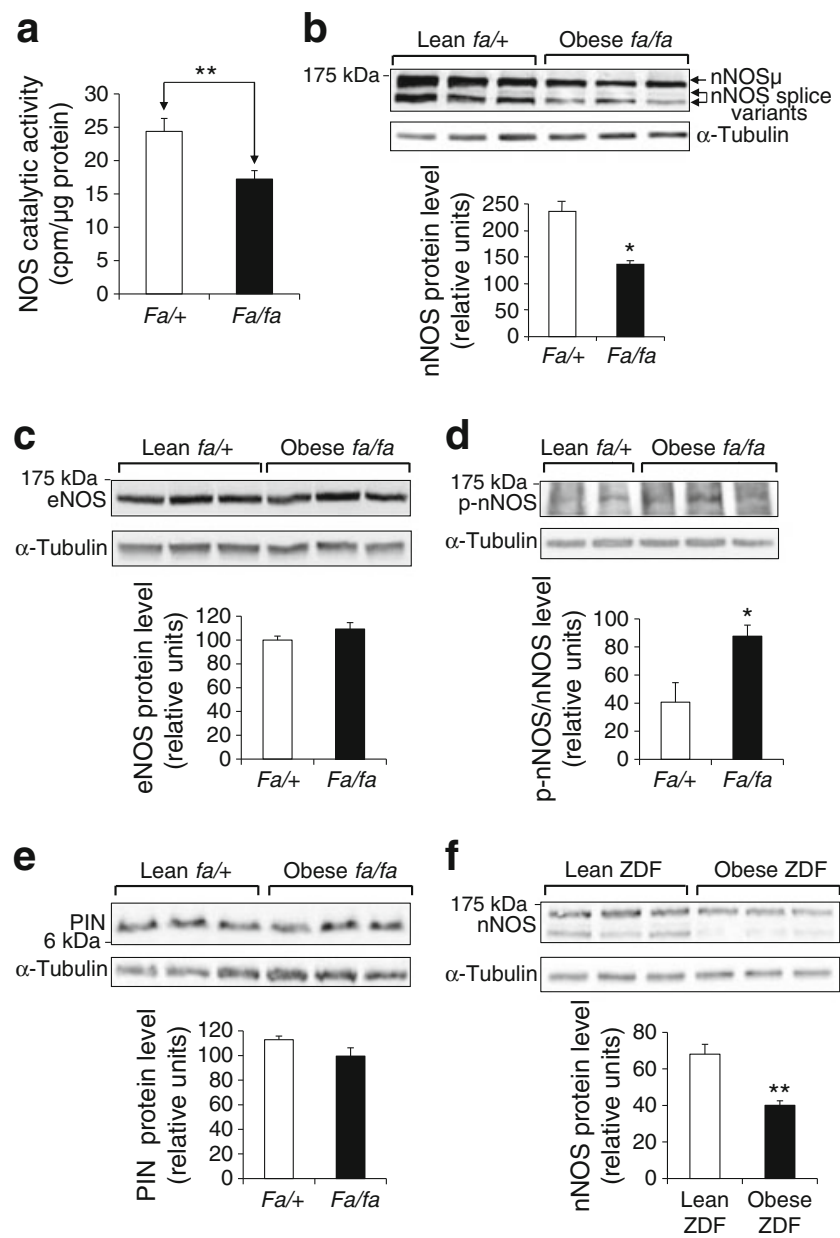

Fig. 1 NOS expression and catalytic activity in the gastrocnemius muscle from Zucker $f a / f a$ and $f a /+$ rats. (a) NOS catalytic activity in muscle from Zucker $f a /+$ vs $f a / f a$ rats $(n=4)$. Expression of (b) nNOS (arrows indicate different splice variants of nNOS), (c) eNOS, (d) p-nNOS on Ser1412 (results are normalised according to the nNOS protein content) and (e) PIN by western blot in $f a /+$ and $f a / f a$ rats. (f) Expression of nNOS by western blot in gastrocnemius muscle from lean and obese ZDF rats. Each western blot is representative of three independent experiments. ${ }^{*} p<0.05 ;{ }^{*} p<0.01$ 
protein content in muscle from $f a / f a$ vs $f a /+$ rats $(p<0.05)$ (Fig. 1b). Interestingly, splice variants of nNOS, including nNOS $\beta$ lacking the N-terminus PDZ domain [16], could also be detected in skeletal muscle (Fig. 1b). By contrast, eNOS expression was found to be unchanged in $f a / f a$ vs $f a /+$ rats (Fig. 1c) and iNOS was not expressed in muscle from $f a / f a$ rats (data not shown). We then looked at nNOS phosphorylation on Ser1412, which has previously been shown to transiently activate the enzyme activity [17], and observed a 2.15 -fold increase in the phospho-nNOS/nNOS ratio in muscle from $f a / f a$ rats (Fig. 1d). Moreover, nNOS has been reported to be modulated by an endogenous protein inhibitor, PIN, that inhibits NO production [18]. However, no change in PIN protein expression could be observed in muscle from $f a / f a$ rats (Fig. 1e). Finally, in gastrocnemius muscles from diabetic ZDF rats, we found, as in Zucker $\mathrm{fa} / \mathrm{fa}$ rats, a $42 \%$ reduction in nNOS expression in obese vs lean rats, but the proportion of each nNOS variant differed between the two animals (Fig. 1f). Thus, NOS defect is related to decreased nNOS expression in insulin-resistant muscle, despite a slight increase in nNOS phosphorylation, probably aimed at compensating its catalytic decline.

Changes in nNOS subcellular localisation We analysed whether nNOS subcellular localisation could be altered in insulin-resistant muscle. As compared with $\mathrm{fa} /+$ rats (Fig. 2a) a drastic decrease in nNOS sarcolemmal immunoreactivity could be observed in fa/fa rats (Fig. 2b), which contrasted with the more slightly reduced nNOS cytoplasmic staining. Subcellular localisation of PIN (Fig. 2d) coincided with that of nNOS in skeletal muscle in $f a /+$ rats (Fig. 2c), and the sarcolemmal localisation of PIN was also found to be decreased in fa/fa rats (Fig. 2f), as for nNOS (Fig. 2e). In addition, expression (data not shown) and localisation of $\alpha 1$-syntrophin, known to anchor nNOS at the level of the sarcolemma [5], were unmodified in muscle from $f a / f a$ rats (compare Fig. 2h, j). Finally, we confirmed changes in nNOS localisation by subcellular fractionation of gastrocnemius muscle. The purity of each fraction was measured by the presence of extracellular-signal-regulated kinase $1 / 2$ (cytoplasmic) and IL-1 receptor type 1 (particulate) (Fig. 2k). We found a $17 \%$ and $58 \%$ decrease in cytoplasmic and particulate nNOS, respectively, in muscle from $f a / f a$ rats (Fig. 2k). The relative level of nNOS in each fraction appeared to be non-comparable because of the experimental conditions.

Evidence for nNOS degradation by the ubiquitin-proteasome pathway Surprisingly, we did not observe any significant variation in $n N o s$ mRNA in $f a / f a$ rats using real-time RT- (reverse transcription) PCR $(n=6)$ (Fig. 3a), suggesting possible nNOS degradation by the ubiquitin proteasome system. In the presence of the proteasome inhibitor MG 132 $(50 \mu \mathrm{mol} / \mathrm{l})$, NOS catalytic activity was found to be unchanged in $f a /+$ rats, whereas in $f a / f a$ rats, it was restored to a level similar to that observed in control rats ( $47 \%$ increase, $p<0.05, n=4$ ) (Fig. 3b). Moreover, MG 132 treatment normalised nNOS protein content in $f a / f a$ rats $(p<0.05)$,
Fig. 2 Subcellular localisation of nNOS, PIN and $\alpha 1$-syntrophin in gastrocnemius muscle sections from Zucker $f a /+$ and $f a / f a$ rats analysed by immunofluorescence. Sections from $f a /+(\mathbf{a}, \mathbf{c}, \mathbf{d}, \mathbf{g}, \mathbf{h})$ and $f a / f a$ $(\mathbf{b}, \mathbf{e}, \mathbf{f}, \mathbf{i}, \mathbf{j})$ rats were labelled for $\operatorname{nNOS}(\mathbf{a}, \mathbf{b}, \mathbf{c}, \mathbf{e}, \mathbf{g}, \mathbf{i}), \operatorname{PIN}(\mathbf{d}, \mathbf{f})$ and $\alpha 1$-syntrophin $(\mathbf{h}, \mathbf{j})$. Scale bars, $50 \mu \mathrm{m}$. (k) Expression of nNOS by western blot in cytoplasmic and particulate fractions of gastrocnemius muscle from $f a /+$ and $f a / f a$ rats. The purity of each fraction was checked by evaluating the presence of extracellular-signalregulated kinase 1/2 (ERK1/2; cytoplasmic fraction, $\mathrm{CF}$ ) and IL-1 receptor type 1 (IL1R1; particulate fraction, $\mathrm{PF})$. $* p<0.05 ; * * * p<0.001$
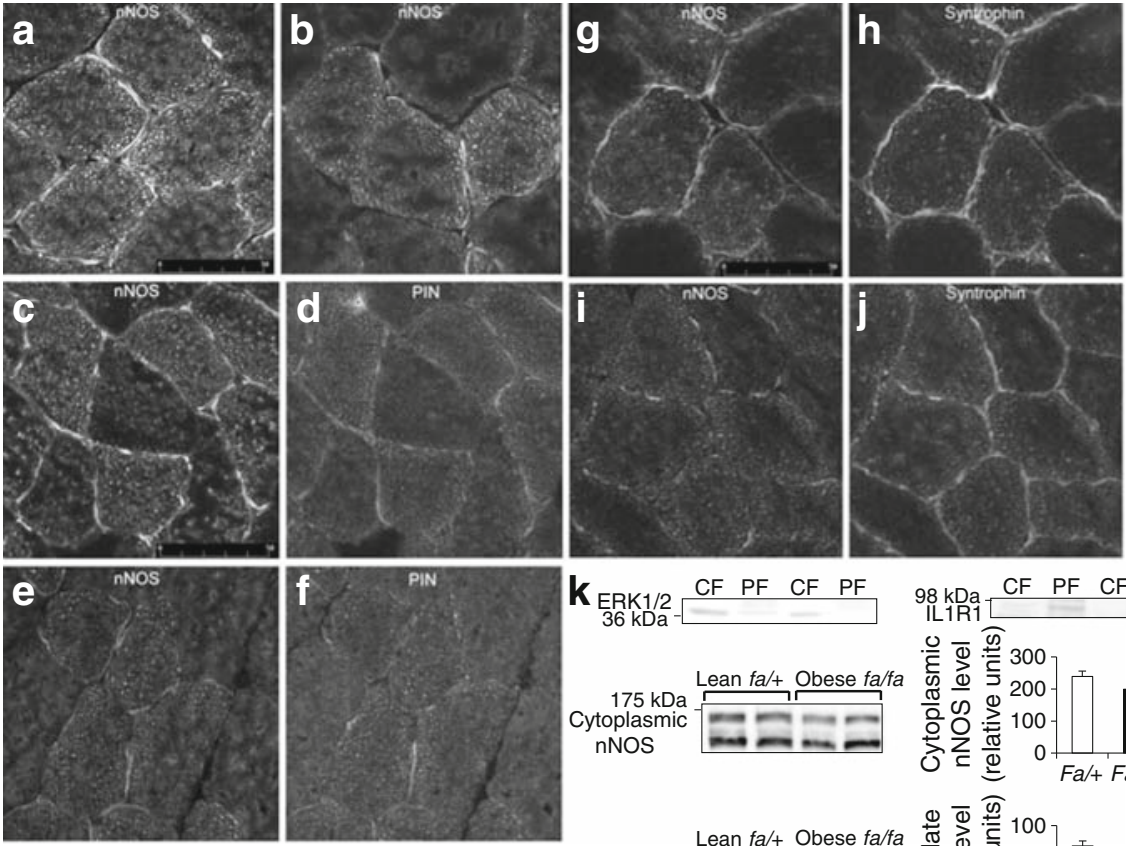
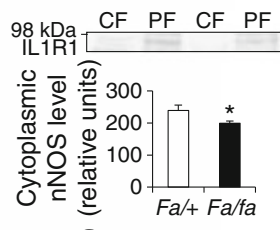

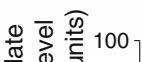

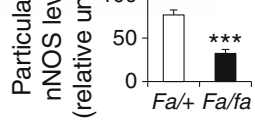


a

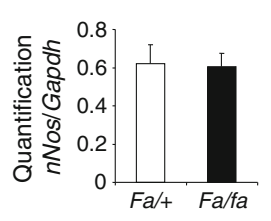

C
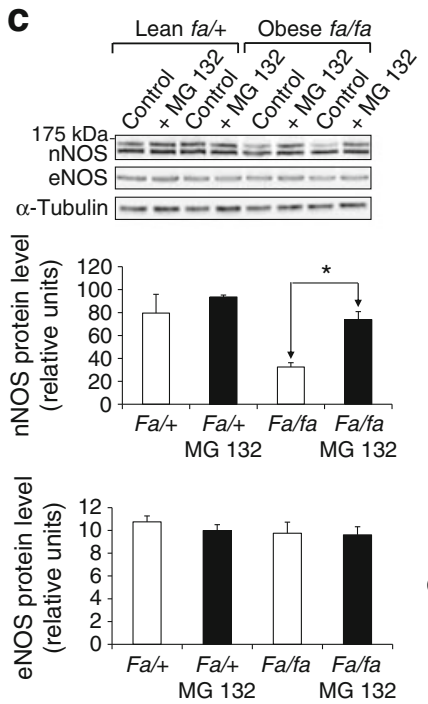

b

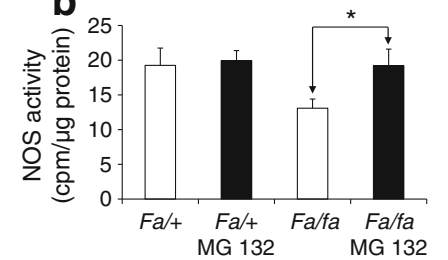

d
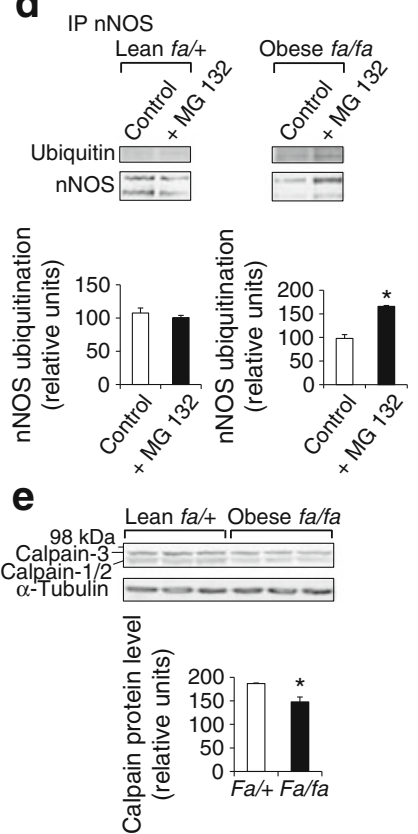

Fig. 3 Evidence for nNOS degradation by the ubiquitin-proteasome pathway in isolated gastrocnemius muscle from Zucker $f a / f a$ vs $f a /+$ rats. (a) Expression of $n N o s$ analysed by real-time RT (reverse transcription)-PCR in $f a /+$ and $f a / f a$ rats $(n=6)$. (b) Effect of the proteasome inhibitor MG $132(50 \mu \mathrm{mol} / \mathrm{l})$ on nNOS catalytic activity in $f a /+$ vs $f a / f a$ rats $(n=4)$. (c) Effect of MG $132(50 \mu \mathrm{mol} / 1)$ on $n N O S$ and eNOS expression in $f a /+$ vs $f a / f a$ rats and relative quantification of the band intensities. (d) Ubiquitination of nNOS in $f a /+$ and $f a / f a$ rats in the presence or absence of MG 132. nNOS was immunoprecipitated and the presence of ubiquitin and nNOS revealed by western blot. (e) Expression of calpains by western blot in $f a /+$ and $f a / f a$ rats. ${ }^{*} p<0.05$

whereas eNOS expression was unchanged by MG 132 (Fig. 3c). As eNOS is also activated by phosphorylation on serine 1177 by Akt [19], we measured eNOS phosphorylation by western blot and found no changes in $f a / f a$ vs $f a /+$ rats (data not shown). Interestingly, the entire nNOS $\mu$ was more subject to proteolytic degradation than the other splice variants lacking the PDZ domain. In addition, using immunoprecipitation, we observed an accumulation of ubiquitinated nNOS protein in muscle from $f a / f a$ as compared with $f a /+$ rats, which was reinforced by treatment with MG 132 (Fig. 3d). Finally, we investigated whether the $\mathrm{Ca}^{2+}$-dependent protease calpain could also be involved in nNOS degradation, as previously described [20]. We found $20 \%$ decreased expression of calpain- 3 and $-1 / 2$ in muscle from $f a / f a$ rats $(p<0.05)$ (Fig. 3e). Overall, our results suggest that the ubiquitin-proteasome system, rather than the protease

calpain, is involved in nNOS degradation in insulin-resistant skeletal muscle.

Effect of nNOS proteasomal degradation blockade on glucose uptake in primary muscle cells In isolated rat muscle, we confirmed decreased insulin sensitivity in $f a / f a$ vs $f a /+$ muscles $\left(-21 \pm 5 \%\right.$, from 3.02 to $\left.2.4 \mathrm{cpm} \mathrm{mg}^{-1} \mathrm{~min}^{-1}\right)$. For further experiments, we isolated satellite cells from gastrocnemius muscle and differentiated them into myotubes to perform glucose-uptake experiments. Basal glucose uptake was reduced by $21 \%$ in muscle cells from $f a / f a$ vs $f a /+$ rats (from $3,003 \pm 245$ to $2,364 \pm 223 \mathrm{cpm} \mathrm{mg}^{-1} \mathrm{~min}^{-1} ; p<0.05$; $n=6$ ), as previously described [21]. In $f a /+$ rats, insulin dose-dependently stimulated glucose uptake at $10 \mathrm{nmol} / 1$ $(+24 \pm 2.2 \% ; p<0.01 ; n=6)$ and $100 \mathrm{nmol} / 1(+44 \pm 6.5 \%$; $p<0.01 ; n=6$ ) (Fig. 4a). Insulin slightly promoted glucose a
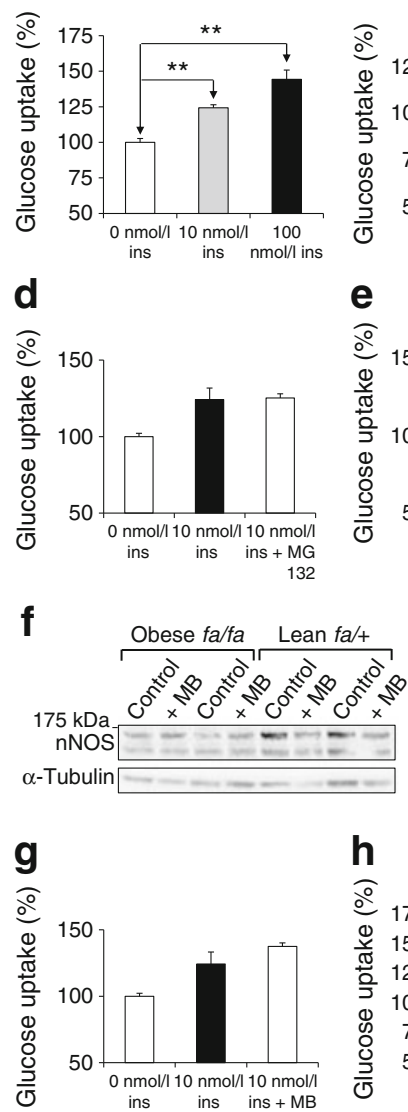
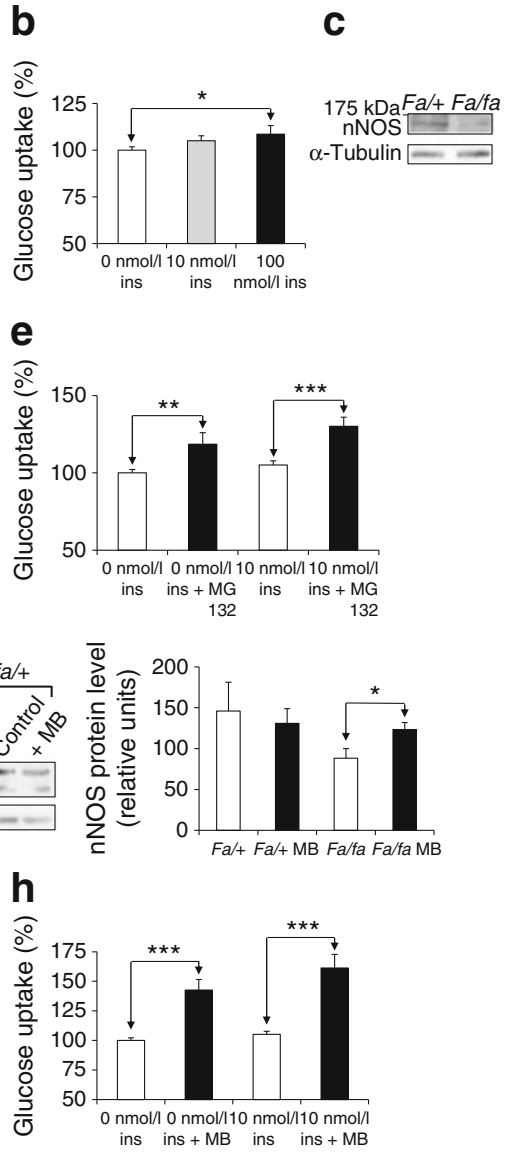

Fig. 4 Effect of nNOS proteasomal degradation blockade on glucose uptake in primary muscle cells from Zucker $f a / f a$ vs $f a /+$ rats. Effect of increasing concentrations of insulin (ins; $0,10,100 \mathrm{nmol} / \mathrm{l}$ ) on glucose uptake in muscle cells from $f a /+$ (a) and $f a / f a$ (b) rats. (c) Expression of nNOS by western blot in primary muscle cells from $f a / t$ and $f a / f a$ rats. Effect of MG $132(50 \mu \mathrm{mol} / \mathrm{l})$ on glucose uptake in muscle cells from $f a /+(\mathbf{d})$ and $f a / f a(\mathbf{e})$ rats, with or without $10 \mathrm{nmol} / 1$ insulin. (f) Effect of Methylene Blue (MB; $10 \mu \mathrm{mol} / \mathrm{l})$ on $\mathrm{nNOS}$ expression in isolated muscle from $f a /+$ vs $f a / f a$ rats. Effect of Methylene Blue on glucose uptake in muscle cells from $f a /+(\mathbf{g})$ and $(\mathbf{h}) f a / f a$ rats, with or without $10 \mathrm{nmol} / 1$ insulin. ${ }^{*} p<0.05 ;{ }^{* *} p<0.01 ;{ }^{* * *} p<0.001$ 
uptake at the $100 \mathrm{nmol} / 1$ concentration in insulin-resistant muscle cells $(+9 \pm 4.6 \% ; p<0.05 ; n=6)$ (Fig. 4b), as observed by others in type 2 diabetic patients [22]. We confirmed that nNOS expression remained reduced in cultured myocytes, as in entire muscles (Fig. 4c). The proteasome inhibitor MG 132 $(50 \mu \mathrm{mol} / \mathrm{l})$ did not modify glucose uptake in $f a /+$ muscle cells $(n=5)$ (Fig. 4d), whereas MG 132 increased glucose uptake by $18 \pm 5 \%(p<0.01 ; n=5)$ and $30 \pm 6 \%(p<0.001$; $n=5$ ), respectively, in the absence and presence of insulin $(10 \mathrm{nmol} / \mathrm{l})$ in $f a / f a$ rats (Fig. 4e). As nNOS degradation is dependent upon the heat-shock protein (Hsp)70 chaperone machinery [23], we also used Methylene Blue, an inhibitor of Hsp70-dependent proteasomal degradation [24], and observed that the inhibitor was able to restore nNOS expression in muscle from fa/fa rats (Fig. 4f). In addition, Methylene Blue $(10 \mu \mathrm{mol} / \mathrm{l})$, which was ineffective in $f a /+$ rats (Fig. 4g), improved basal and insulin-stimulated (10 nmol/l) glucose uptake by $42 \pm 6 \%$ and $61 \pm 11.5 \%$, respectively, in $f a / f a$ muscle cells $(p<0.001 ; n=5)$ (Fig. 4h).

Finally, several lysine residues have been predicted to be potential nNOS ubiquitination sites, using the UbPred program (www.ubpred.org) [25]. As suggested by Fig. 3c, the main site is probably localised in the N-terminus of the enzyme. To specifically counteract nNOS proteasomal degradation, we designed an inhibitory peptide targeting a high confidence ubiquitination site located at Lys131 of rat nNOS and coupled it to the Tat sequence, to render it cell permeant. Indeed, inhibitory peptides have been shown to specifically counteract the proteasomal degradation of a target protein [26]. Moreover, cell-penetrating peptides have been shown to be easily captured in primary cardiomyocytes in vitro [27]. In muscles cells from $f a / f a$ rats, the Tat peptide increased nNOS expression by $84 \%$ (Fig. 5a) and improved glucose uptake in both basal and insulin-stimulated $(10 \mathrm{nmol} / \mathrm{l})$ conditions $(+11 \pm 3.5 \% ; p<0.01 ; n=7)$ (Fig. 5b). Thus, we conclude that specifically counteracting nNOS proteasomal
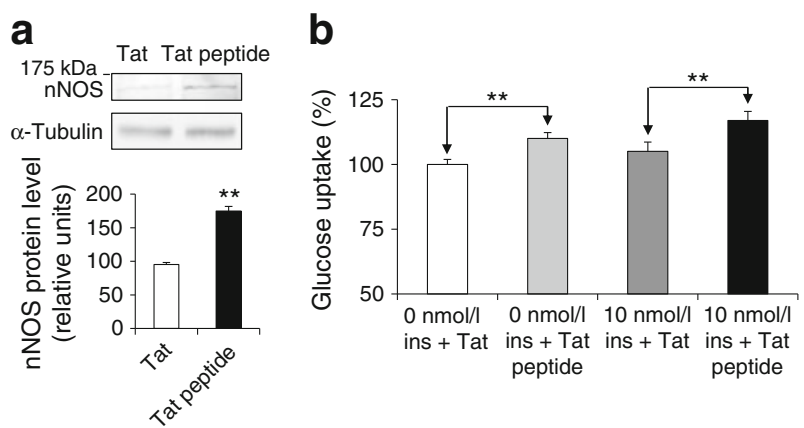

Fig. 5 Effect of nNOS restoration by the inhibitory Tat peptide on glucose uptake in primary muscle cells from Zucker $f a / f a$ rats. (a) Western blot analysis of nNOS expression with cell-permeant inhibitory Tat peptide or Tat alone $(20 \mu \mathrm{mol} / \mathrm{l})$. (b) Effect of Tat peptide on glucose uptake in cells from $f a / f a$ rats, with or without $10 \mathrm{nmol} / 1$ insulin (ins). $* * p<0.01$ degradation improves glucose uptake in insulin-resistant muscle cells.

Effect of nNOS restoration on glucose uptake in primary muscle cells We first checked whether muscle cells from $\mathrm{fa} / \mathrm{fa}$ rats retain their sensitivity to sodium nitroprusside (SNP), as previously described [21]. SNP (1 mmol/l) enhanced basal $(+29 \pm 4 \% ; p<0.001 ; n=5)$ and insulin-stimulated $(10 \mathrm{nmol} / 1)(+44 \pm 6 \% ; p<0.001 ; n=5)$ glucose uptake in myocytes from $f a /+$ rats (Fig. 6a). Cells from insulin-resistant $f a / f a$ rats remained sensitive to SNP in both basal $(+30 \pm 6 \%$; $p<0.001 ; n=5)$ and insulin-stimulated $(10 \mathrm{nmol} / \mathrm{l})(+48 \pm 7 \%$; $p<0.001 ; n=5)$ glucose uptake (Fig. 6b). When muscle cells from $f a / f a$ rats were transfected with an expression vector coding for $\mathrm{nNOS} \alpha$, nNOS expression was increased threefold (Fig. 6c) and glucose uptake improved by $17 \pm 8 \%(p<0.05$; $n=5)$ and $28 \pm 9.8 \%(p<0.001 ; n=5)$ in basal and insulin-
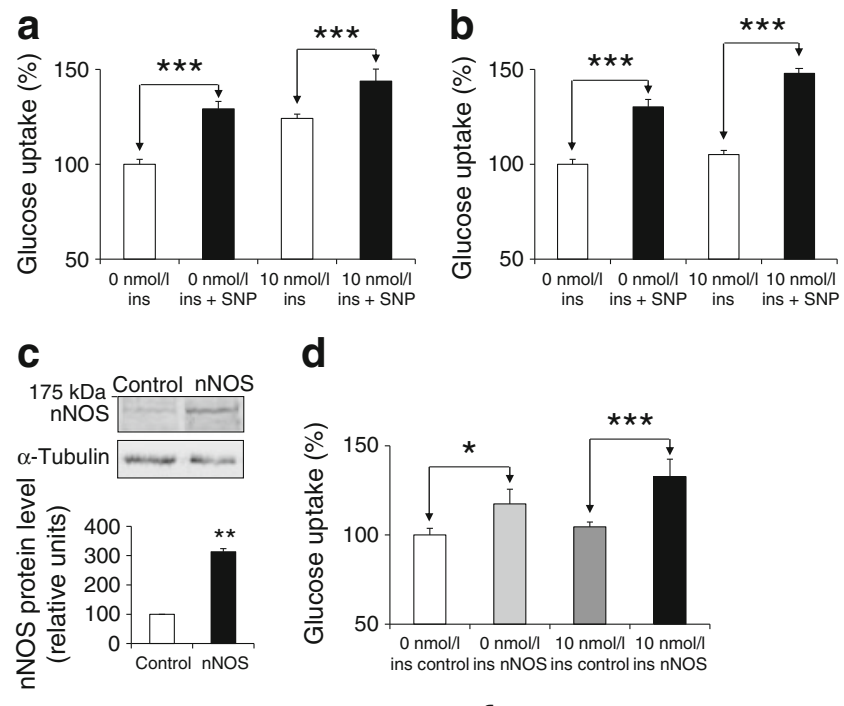

d
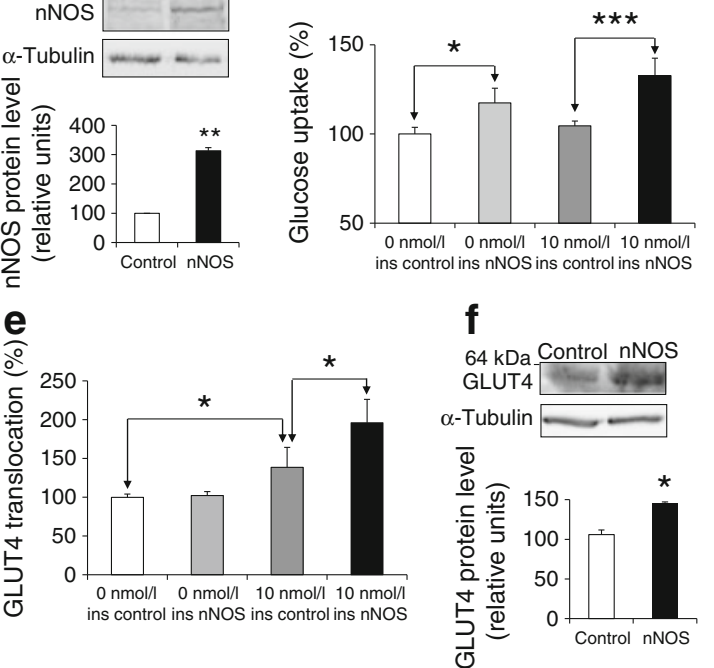

Fig. 6 Effect of nNOS $\alpha$ restoration by overexpression on glucose uptake and GLUT4 translocation in primary muscle cells from Zucker fa/fa rats. Effect of SNP (1 mmol/l) on glucose uptake in $f a /+$ (a) and $f a / f a$ (b) rats, with or without $10 \mathrm{nmol} / \mathrm{l}$ insulin (ins). (c) Western blot analysis of nNOS overexpression after transfection with $n N o s \alpha$ cDNA (nNOS) or an empty vector (control). (d) Effect of nNOS $\alpha$ overexpression (nNOS) on glucose uptake in muscle cells from $f a / f a$ rats, with or without $10 \mathrm{nmol} / \mathrm{l}$ insulin. (e) Basal or insulin $(10 \mathrm{nmol} / \mathrm{l})$ effects on GLUT4 translocation in muscle cells from $f a / f a$ rats transfected with $n N o s \alpha$ cDNA (nNOS) or an empty vector (control). (f) Western blot analysis of GLUT4 expression after transfection with $n N o s \alpha$ cDNA (nNOS) or an empty vector (control). ${ }^{*} p<0.05 ; * * p<0.01 ; * * * p<0.001$ 
stimulated (10 nmol/l) conditions, respectively (Fig. 6d). Despite the use of $n N O S \alpha$ instead of nNOS $\mu$ in our overexpression experiments, we propose that restoration of nNOS expression was able to improve insulin-stimulated glucose utilisation in insulin-resistant skeletal muscle.

Improvement of GLUT4 translocation by nNOS restoration We first found no differences in GLUT4 expression in muscle from $f a / f a$ and $f a /+$ rats (data not shown). Then, to address the mechanisms by which nNOS restoration improves glucose uptake in muscle cells from $\mathrm{fa} / \mathrm{fa}$ rats, we measured GLUT4 translocation and found a $96 \%$ increase in GLUT4 transport $(p<0.05, n=3)$ when nNOS was overexpressed in the presence of insulin, but not in basal conditions (Fig. 6e). Interestingly, $10 \mathrm{nmol} / \mathrm{l}$ insulin, which was unable to enhance glucose uptake (see Fig. 4b), stimulated GLUT4 transport by $38 \%(p<0.05, n=3)$. NO has also been shown to modulate GLUT4 expression in L6 myotubes [28], and we found a 38\% increase in GLUT4 protein level in muscle cells from $f a / f a$ rats overexpressing nNOS $(p<0.05)$ (Fig. 6f).

Involvement of a ternary complex between nNOS, Hsp70 and CHIP in nNOS proteolytic degradation nNOS is known to interact with two chaperones, Hsp90 and Hsp70, which oppositely affect nNOS stability: Hsp90 participates in inhibition and Hsp70 in stimulation of nNOS ubiquitination [29]. Proteasomal degradation of nNOS requires ubiquitination by the chaperone-dependent E3 ubiquitin ligase, CHIP [23]. Hsp70 and CHIP were found to be expressed in skeletal muscle. No difference in Hsp70 expression could be observed in muscles from $\mathrm{fa} /+$ and $\mathrm{fa} / \mathrm{fa}$ rats (Fig. 7a); in contrast, CHIP expression was higher in $f a / f a$ vs $f a /+$ rats $(p<0.01)$ (Fig. 7b). Using immunoprecipitation experiments, we found an nNOS-Hsp70-CHIP ternary complex in skeletal muscle. Most importantly, greater amounts of nNOS $(p<0.05)$ and Hsp70 $(p<0.05)$ could be detected when CHIP was immunoprecipitated from $\mathrm{fa} / \mathrm{fa}$ rat muscles (Fig. 7c). Hence, we conclude that nNOS proteasomal degradation in the insulin-resistant skeletal muscle involves both Hsp70 and the ubiquitin ligase CHIP.

\section{Discussion}

The $n N O S \mu$ isoform plays a key role in the regulation of contractile force and glucose utilisation in skeletal muscle $[6,7]$. Early studies suggested the presence of defects in the nNOS signalling pathway in insulin-resistant muscle. More precisely, skeletal muscles from Zucker $f a / f a$ rats have been reported to display decreased NOS catalytic activity [11]. a

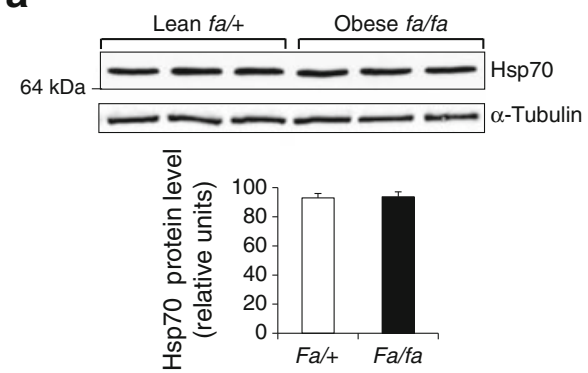

b

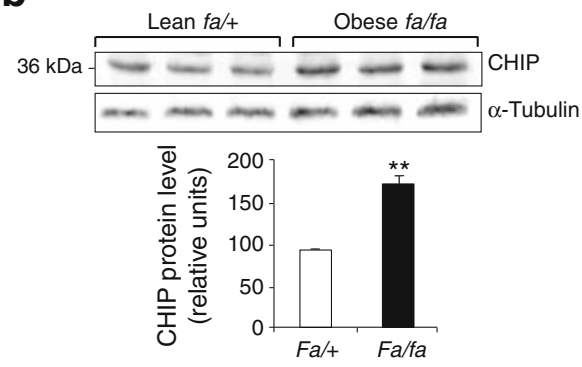

C
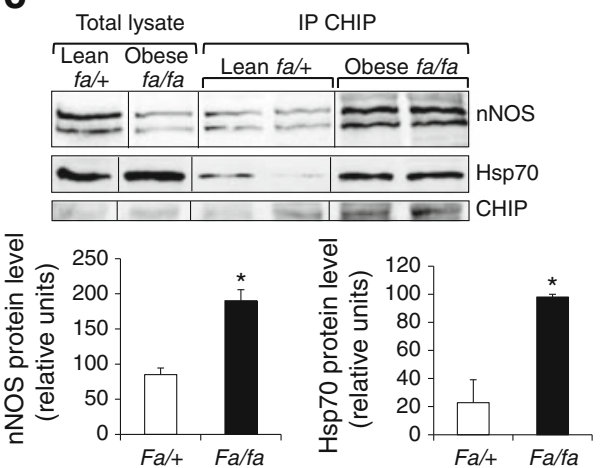

Fig. 7 Evidence for a ternary complex between nNOS, Hsp70 and CHIP in gastrocnemius muscle from Zucker $f a / f a$ vs $f a /+$ rats. Expression of Hsp70 (a) and CHIP (b) by western blot in muscle extracts from $f a /+$ and $\mathrm{fa} / \mathrm{fa}$ rats, and relative quantification of the band intensities. (c) Co-immunoprecipitation of CHIP with Hsp70 and nNOS in muscles from $\mathrm{fa} /+$ and $f a / f a$ rats. CHIP was immunoprecipitated (IP) and the presence of Hsp70 and nNOS was revealed by western blot. Each western blot is representative of three independent experiments. ${ }^{*} p<0.05 ;{ }^{*} p<0.01$

Impaired basal and insulin-stimulated muscle NOS activity has also been described in insulin-resistant muscle from individuals with type 2 diabetes [30]. These observations prompted us to study the mechanisms responsible for an nNOS defect in insulin-resistant muscle, using the Zucker falfa rat as a model.

The decreased nNOS expression we observed in Zucker $f a / f a$ rats partly accounts for the impaired nNOS catalytic activity. A decrease in skeletal muscle nNOS expression occurs in a broad panel of glucose homeostasis disorders; it has been found in ZDF as well as in Goto-Kakizaki diabetic rats [31], and in prediabetic and type 2 diabetic individuals $[32,33]$. 
Concerning eNOS, this isoform has been shown to be expressed in endothelial cells and some skeletal muscle fibres [3]. Even though the endothelium-dependent vasodilator response is impaired in insulin-resistant muscle [34], we found it unchanged in our study in Zucker fa/fa rat muscle, despite previous conflicting data $[35,36]$.

Proteolytic degradation of NOS can be considered as a regulatory mechanism aimed at controlling NOS protein quality. In insulin-resistant skeletal muscle, we found that nNOS was actively degraded by the proteasome [37], as demonstrated by the presence of high amounts of ubiquitinated nNOS and by the effect of the inhibitor MG 132 , which was able to restore nNOS expression and catalytic activity to a normal level. We also detected lower amounts of SDS-resistant dimers in $f a / f a$ vs $f a /+$ skeletal muscle (data not shown), which might favour increased susceptibility to proteasomal degradation; indeed, dimerisation is known to protect nNOS from proteolysis [38]. The specific signals targeting nNOS to the proteasome have not been clearly identified. A recent study reported that Lys 739 in the calmodulin-binding domain is a site for ubiquitination of nNOS in vitro and participates in the CHIP-dependent degradation of nNOS in HEK293 cells [39]. Based on the UbPred prediction [25], we also identified Lys131 as a potential ubiquitination site, in accordance with our observation that the entire nNOS undergoes more degradation than nNOS splice variants that lack the PDZ domain (Fig. 3c). Using a cell-permeant peptide counteracting Lys 131 ubiquitination, we partially restored nNOS expression, and thereby confirmed the involvement of the N-terminal part of the enzyme in its proteasomal degradation. Nonetheless, other ubiquitination sites that are present in all nNOS variants (e.g. Lys739 [39]) might also participate in degradation of the enzyme, but with less important turnover.

Proteins assigned to degradation are labelled with a multiubiquitin chain and then targeted to the $26 \mathrm{~S}$ proteasome. Among the ubiquitination enzymes, CHIP is a U-boxcontaining E3 ubiquitin ligase that binds to the chaperones Hsp70 and Hsp90 [40]. It has been demonstrated that CHIP ubiquitinates nNOS and that the quantity of nNOS-ubiquitin conjugates is greatly enhanced upon the interaction of CHIP with nNOS-bound Hsp70 [23]. In contrast, binding of Hsp90 to nNOS has been reported to enhance the enzyme's catalytic activity [41], together with calmodulin-dependent inhibition of its ubiquitination [29]. We showed that CHIP and Hsp70 are both present in skeletal muscle. Hsp70 expression was unchanged and CHIP expression was increased in $f a / f a$ rats, a finding that could relate to the enhanced proteolysis and muscle wasting observed in insulin-resistant skeletal muscle [42]. Indeed, increased CHIP-dependent proteasomal activity occurred in $f a / f a$ muscle, as pharmacological blockade with MG 132 and, more interestingly, with Methylene Blue highly improved glucose uptake in insulin-resistant muscle but not in insulin-sensitive tissue. Furthermore, our immunoprecipitation experiments confirmed the presence of a ternary complex between nNOS, Hsp70 and CHIP in skeletal muscle. Most importantly, the greater association between the three partners in insulin-resistant muscle is likely to account for the increased proteolytic degradation of nNOS.

In Zucker rats, we confirmed that nNOS is strongly associated with the sarcolemma of muscle fibres, as already described by others $[4,43]$. We also detected a more punctuated staining in the sarcoplasm, probably corresponding to nNOS localised in intracellular organelles such as the Golgi complex [16]. An important observation is the strong decrease in sarcolemmal localisation of nNOS in skeletal muscle from Zucker $f a / f a$ rats. Such a change has also been shown to occur for nNOS in muscle fibres from patients with Duchenne muscular dystrophy [44] and in the GotoKakizaki rat model of type 2 diabetes [31]. From a pathophysiological point of view, alterations in the $\mathrm{nNOS}$ signalling pathway resulting from nNOS mislocalisation might be involved in muscle fatigue and atrophy intervening in insulin-resistant skeletal muscle [45, 46]. Concerning the expression of the protein inhibitor of nNOS, PIN, originally cloned in rat brain [18], and identified in ventilatory muscles [43] and pancreatic beta cells [47], we found levels of PIN to be almost unchanged in insulin-resistant muscles. In addition, PIN sarcolemmal localisation decreased in line with that of nNOS, pointing to a minor role of PIN in nNOS alterations.

Of major interest is the observation that restoration of nNOS expression by blocking nNOS degradation or by nNOS overexpression was able to improve insulin-stimulated glucose uptake in primary muscle cells from $f a / f a$ rats. We are aware of the potential limitations associated with the use of $n N O S \alpha$ instead of $n N O S \mu$ in these overexpression experiments, despite the fact that both isoforms share the same ubiquitination sites and display identical catalytic activity [4]. Very interestingly, $f a / f a$ muscle cells retained their sensitivity to NO, as shown by the stimulating effect of the NO donor, SNP, independently of the presence of insulin. Such a positive effect of NO has also been found in insulin-resistant muscle cells derived from individuals with type 2 diabetes [21]. In addition, our observations, as those of several other groups, support the view that NO controls glucose transport via insulin-independent signalling pathways [48, 49], including activation of cGMP-dependent protein kinase $[50,51]$ and AMP-activated protein kinase- $\alpha 1[48,52]$. Concerning the possible mechanisms involved, we found that restoration of endogenous nNOS in insulin-resistant muscle cells greatly improved translocation of GLUT4 at the plasma membrane in the presence of insulin, but not in basal conditions. This implies that normal nNOS activity/expression could also improve the intrinsic activity of GLUT4, as previously suggested [53], despite no consensus view in the literature. In addition, nNOS restoration increased GLUT4 expression, 
as previously described in L6 myocytes [28], which may participate at the greater level of the transporter observed at the plasma membrane. We also found that insulin moderately stimulated GLUT4 translocation in muscles cells from $f a / f a$ rats, without any improvement in glucose uptake. Such a finding suggests that insulin resistance in $f a / f a$ rats could, at least in part, also result from a defect in GLUT4 activation.

In conclusion, insulin-resistant skeletal muscle from Zucker $f a / f a$ rats displayed decreased nNOS protein expression and mislocalisation, which could both account for the impaired nNOS catalytic activity. The loss of nNOS was caused by increased degradation of the enzyme by the ubiquitin-proteasome pathway, mediated by a direct interaction with Hsp70 and CHIP. Finally, nNOS restoration in insulin-resistant muscles should be considered as a potential new approach to improve muscle glucose homeostasis.

Acknowledgements We thank M. Manteghetti, from the Centre for Pharmacology and Innovation in Diabetes, for her technical contribution.

Funding ADL was supported by a grant from the Fondation pour la Recherche Médicale and Association pour la Recherche sur le Diabète.

Duality of interest The authors declare that there is no duality of interest associated with this manuscript.

Contribution statement All authors contributed to the conception, design or analysis and interpretation of data, to drafting and revising the manuscript, and to approval of the final version of the manuscript.

\section{References}

1. Moncada S, Palmer RM, Higgs EA (1991) Nitric oxide: physiology, pathophysiology, and pharmacology. Pharmacol Rev 43:109-142

2. Frandsen U, Lopez-Figueroa M, Hellsten Y (1996) Localization of nitric oxide synthase in human skeletal muscle. Biochem Biophys Res Commun 227:88-93

3. Kobzik L, Stringer B, Balligand JL, Reid MB, Stamler JS (1995) Endothelial type nitric oxide synthase in skeletal muscle fibers: mitochondrial relationships. Biochem Biophys Res Commun 211: 375-381

4. Silvagno F, Xia H, Bredt DS (1996) Neuronal nitric-oxide synthase$\mathrm{mu}$, an alternatively spliced isoform expressed in differentiated skeletal muscle. J Biol Chem 271:11204-11208

5. Lapidos KA, Kakkar R, McNally EM (2004) The dystrophin glycoprotein complex: signaling strength and integrity for the sarcolemma. Circ Res 94:1023-1031

6. Stamler JS, Meissner G (2001) Physiology of nitric oxide in skeletal muscle. Physiol Rev 81:209-237

7. McConell GK, Rattigan S, Lee-Young RS, Wadley GD, Merry TL (2012) Skeletal muscle nitric oxide signaling and exercise: a focus on glucose metabolism. Am J Physiol Endocrinol Metab 303:E301E307

8. Eriksson J, Koranyi L, Bourey R et al (1992) Insulin resistance in type 2 (non-insulin-dependent) diabetic patients and their relatives is not associated with a defect in the expression of the insulinresponsive glucose transporter (GLUT-4) gene in human skeletal muscle. Diabetologia 35:143-147
9. Bjornholm M, Zierath JR (2005) Insulin signal transduction in human skeletal muscle: identifying the defects in type II diabetes. Biochem Soc Trans 33:354-357

10. Shankar RR, Wu Y, Shen HQ, Zhu JS, Baron AD (2000) Mice with gene disruption of both endothelial and neuronal nitric oxide synthase exhibit insulin resistance. Diabetes 49:684-687

11. Young ME, Leighton B (1998) Evidence for altered sensitivity of the nitric oxide/cGMP signalling cascade in insulin-resistant skeletal muscle. Biochem J 329:73-79

12. Phillips MS, Liu Q, Hammond HA et al (1996) Leptin receptor missense mutation in the fatty Zucker rat. Nature Genet 13:18-19

13. Allen RE, Temm-Grove CJ, Sheehan SM, Rice G (1997) Skeletal muscle satellite cell cultures. Methods Cell Biol 52:155-176

14. Machida S, Spangenburg EE, Booth FW (2004) Primary rat muscle progenitor cells have decreased proliferation and myotube formation during passages. Cell Prolif 37:267-277

15. Brooks H, Lebleu B, Vives E (2005) Tat peptide-mediated cellular delivery: back to basics. Adv Drug Deliv Rev 57:559-577

16. Percival JM, Anderson KN, Huang P, Adams ME, Froehner SC (2010) Golgi and sarcolemmal neuronal NOS differentially regulate contraction-induced fatigue and vasoconstriction in exercising mouse skeletal muscle. J Clin Invest 120:816-826

17. Rameau GA, Tukey DS, Garcin-Hosfield ED et al (2007) Biphasic coupling of neuronal nitric oxide synthase phosphorylation to the NMDA receptor regulates AMPA receptor trafficking and neuronal cell death. J Neurosci 27:3445-3455

18. Jaffrey SR, Snyder SH (1996) PIN: an associated protein inhibitor of neuronal nitric oxide synthase. Science 274:774-777

19. Dimmeler S, Fleming I, Fisslthaler B et al (1999) Activation of nitric oxide synthase in endothelial cells by Akt-dependent phosphorylation. Nature 399:601-605

20. Laine R, de Montellano PR (1998) Neuronal nitric oxide synthase isoforms alpha and mu are closely related calpain-sensitive proteins. Mol Pharmacol 54:305-312

21. Henstridge DC, Drew BG, Formosa MF et al (2009) The effect of the nitric oxide donor sodium nitroprusside on glucose uptake in human primary skeletal muscle cells. Nitric Oxide 21:126-131

22. Shemyakin A, Salehzadeh F, Esteves Duque-Guimaraes D et al (2011) Endothelin-1 reduces glucose uptake in human skeletal muscle in vivo and in vitro. Diabetes 60:2061-2067

23. Peng HM, Morishima Y, Jenkins GJ et al (2004) Ubiquitylation of neuronal nitric-oxide synthase by CHIP, a chaperone-dependent E3 ligase. J Biol Chem 279:52970-52977

24. Wang AM, Morishima Y, Clapp KM et al (2010) Inhibition of hsp70 by methylene blue affects signaling protein function and ubiquitination and modulates polyglutamine protein degradation. J Biol Chem 285:15714-15723

25. Radivojac P, Vacic V, Haynes C et al (2010) Identification, analysis, and prediction of protein ubiquitination sites. Proteins Struct Funct Bioinforma 78:365-380

26. Costes S, Vandewalle B, Tourrel-Cuzin C et al (2009) Degradation of cAMP-responsive element-binding protein by the ubiquitinproteasome pathway contributes to glucotoxicity in beta-cells and human pancreatic islets. Diabetes 58:1105-1115

27. Boisguerin P, Redt-Clouet C, Franck-Miclo A et al (2011) Systemic delivery of $\mathrm{BH} 4$ anti-apoptotic peptide using CPPs prevents cardiac ischemia-reperfusion injuries in vivo. J Control Release 156: $146-153$

28. Lira VA, Soltow QA, Long JH, Betters JL, Sellman JE, Criswell DS (2007) Nitric oxide increases GLUT4 expression and regulates AMPK signaling in skeletal muscle. Am J Physiol Endocrinol Metab 293:E1062-E1068

29. Peng HM, Morishima Y, Clapp KM, Lau M, Pratt WB, Osawa Y (2009) Dynamic cycling with Hsp90 stabilizes neuronal nitric oxide synthase through calmodulin-dependent inhibition of ubiquitination. Biochemistry 48:8483-8490 
30. Kashyap SR, Roman LJ, Lamont J et al (2005) Insulin resistance is associated with impaired nitric oxide synthase activity in skeletal muscle of type 2 diabetic subjects. J Clin Endocrinol Metab 90: $1100-1105$

31. Mulvey C, Harno E, Keenan A, Ohlendieck K (2005) Expression of the skeletal muscle dystrophin-dystroglycan complex and syntrophin-nitric oxide synthase complex is severely affected in the type 2 diabetic Goto-Kakizaki rat. Eur J Cell Biol 84: $867-883$

32. Bradley SJ, Kingwell BA, Canny BJ, McConell GK (2007) Skeletal muscle neuronal nitric oxide synthase micro protein is reduced in people with impaired glucose homeostasis and is not normalized by exercise training. Metab Clin Exp 56:1405-1411

33. Krause M, Rodrigues-Krause J, O'Hagan C et al (2012) Differential nitric oxide levels in the blood and skeletal muscle of type 2 diabetic subjects may be consequence of adiposity: a preliminary study. Metabolism 61:1528-1537

34. Avogaro A, Piarulli F, Valerio A et al (1997) Forearm nitric oxide balance, vascular relaxation, and glucose metabolism in NIDDM patients. Diabetes 46:1040-1046

35. Torres SH, de Sanctis JB, de L Briceño M, Hernandez N, Finol HJ (2004) Inflammation and nitric oxide production in skeletal muscle of type 2 diabetic patients. J Endocrinol 181:419-427

36. Kraus RM, Houmard JA, Kraus WE et al (2012) Obesity, insulin resistance, and skeletal muscle nitric oxide synthase. J Appl Physiol 113:758-765

37. Bender AT, Silverstein AM, Demady DR et al (1999) Neuronal nitricoxide synthase is regulated by the Hsp90-based chaperone system in vivo. J Biol Chem 274:1472-1478

38. Dunbar AY, Kamada Y, Jenkins GJ, Lowe ER, Billecke SS, Osawa Y (2004) Ubiquitination and degradation of neuronal nitric-oxide synthase in vitro: dimer stabilization protects the enzyme from proteolysis. Mol Pharmacol 66:964-969

39. Clapp KM, Peng HM, Jenkins GJ et al (2012) Ubiquitination of neuronal nitric-oxide synthase in the calmodulin-binding site triggers proteasomal degradation of the protein. J Biol Chem 287:4260142610

40. Ballinger CA, Connell P, Wu Y et al (1999) Identification of CHIP, a novel tetratricopeptide repeat-containing protein that interacts with heat shock proteins and negatively regulates chaperone functions. Mol Cell Biol 19:4535-4545
41. Bender AT, Demady DR, Osawa Y (2000) Ubiquitination of neuronal nitric-oxide synthase in vitro and in vivo. J Biological Chem 275: 17407-17411

42. Combaret L, Taillandier D, Attaix D (2001) Nutritional and hormonal control of protein breakdown. Am J Kidney Dis 37:S108-S111

43. Guo Y, Greenwood MT, Petrof BJ, Hussain SN (1999) Expression and regulation of protein inhibitor of neuronal nitric oxide synthase in ventilatory muscles. Am J Respir Cell Mol Biol 20:319-326

44. Guo Y, Petrof BJ, Hussain SN (2001) Expression and localization of protein inhibitor of neuronal nitric oxide synthase in Duchenne muscular dystrophy. Muscle Nerve 24:1468-1475

45. Halvatsiotis P, Short KR, Bigelow M, Nair KS (2002) Synthesis rate of muscle proteins, muscle functions, and amino acid kinetics in type 2 diabetes. Diabetes 51:2395-2404

46. Kobayashi YM, Rader EP, Crawford RW et al (2008) Sarcolemmalocalized nNOS is required to maintain activity after mild exercise. Nature 456:511-515

47. Lajoix AD, Badiou S, Peraldi-Roux S et al (2006) Protein inhibitor of neuronal nitric oxide synthase (PIN) is a new regulator of glucoseinduced insulin secretion. Diabetes 55:3279-3288

48. Etgen GJ Jr, Fryburg DA, Gibbs EM (1997) Nitric oxide stimulates skeletal muscle glucose transport through a calcium/contraction- and phosphatidylinositol-3-kinase-independent pathway. Diabetes 46: 1915-1919

49. Higaki Y, Hirshman MF, Fujii N, Goodyear LJ (2001) Nitric oxide increases glucose uptake through a mechanism that is distinct from the insulin and contraction pathways in rat skeletal muscle. Diabetes 50:241-247

50. Young ME, Leighton B (1998) Fuel oxidation in skeletal muscle is increased by nitric oxide/cGMP - evidence for involvement of cGMP-dependent protein kinase. FEBS Lett 424:79-83

51. Lau KS, Grange RW, Isotani E et al (2000) nNOS and eNOS modulate cGMP formation and vascular response in contracting fast-twitch skeletal muscle. Physiol Genomics 2:21-27

52. Deshmukh AS, Long YC, de Castro Barbosa T et al (2010) Nitric oxide increases cyclic GMP levels, AMP-activated protein kinase (AMPK) alpha1-specific activity and glucose transport in human skeletal muscle. Diabetologia 53:1142-1150

53. Antonescu CN, Thong FSL, Niu W, Karnieli E, Klip A (2005) To be or not to be: regulation of the intrinsic activity of GLUT4. Curr Med Chem Immunol Endocr Metab Agents 5:175-187 